Bull. Korean Math. Soc. 51 (2014), No. 2, pp. 437-442

http://dx.doi.org/10.4134/BKMS.2014.51.2.437

\title{
LOCAL COMPLETENESS, LOWER SEMI CONTINUOUS FROM ABOVE FUNCTIONS AND EKELAND'S PRINCIPLE
}

\author{
Carlos Bosch and René leal
}

\begin{abstract}
In this paper we prove Ekeland's variational principle in the setting of locally complete spaces for lower semi continuous functions from above and bounded below. We use this theorem to prove Caristi's fixed point theorem in the same setting and also for lower semi continuous functions.
\end{abstract}

\section{Introduction}

The lower semi-continuous functions have been widely used to find solutions to some minimization problems. Using this functions the fundamental idea of the variational principle due to I. Ekeland [6] is to assign to a minimization problem a slightly perturbed problem having a solution which is at the same time an approximate solution to the original problem. This localization property is very useful and explains the importance of the result. Since the discovery of the Ekeland's variational principle there have also appeared many extensions or equivalent formulations of the principle $[1,2,3,4,6,7,8,9,12,13,14]$. The results of this paper extend and generalize many results appearing recently in the literature. Here we will use the more general concept of lower semicontinuity from above introduced by Y. Chen, Y. J. Cho and L. Yang [5] in the setting of locally complete spaces to get Ekeland's variational principle and some equivalence Caristi's type theorem..

\section{Preliminaries}

Throughout this paper $(E, \tau)$ will denote a Hausdorff locally convex space (briefly locally convex space) with topology $\tau$, generated by a family of seminorms $\left\{\rho_{\alpha}: \alpha \in \Lambda\right\}$ with $\Lambda$ a set of indices. A disk $B$ in $E$ is a closed, bounded and absolutely convex set. We denote by $\left(E_{B}, \rho_{B}\right)$ the linear span of $B$ endowed with the topology defined by the Minkowski functional associated with

Received October 12, 2012; Revised August 3, 2013.

2010 Mathematics Subject Classification. 49J53, 47H10, 46N10.

Key words and phrases. locally complete spaces, lower semi-continuity from above, variational principle, fixed point, minimization, equilibrium.

The first author was partially supported by the Asociación Mexicana de Cultura A.C. 
$B$. When $B$ is bounded $\rho_{B}$ is a norm, and the norm topology is finer than the topology inherited from E. If $\left(E_{B}, \rho_{B}\right)$ is a Banach space we say that $B$ is a Banach disk. We say that $E$ is a locally complete locally convex space (briefly locally complete space) if each closed, bounded disk is a Banach disk. There are many examples of locally complete spaces, in fact every sequential complete space is locally complete. Typical examples of locally complete spaces arise in the following way. Let $(E,\|\cdot\|)$ be a Banach space and $\sigma\left(E, E^{\prime}\right)$ be the weak topology in $E$ then $\left(E, \sigma\left(E, E^{\prime}\right)\right)$ is a locally complete space and not sequentially complete. For metrizable locally convex spaces these concepts are equivalent.

The class $\Phi$ of perturbations we will use, is defined as the family of functions $\varphi:[0, \infty) \rightarrow[0, \infty)$ which are subadditive, strictly increasing, continuous, $\varphi(0)=0$, and $\lim _{x \rightarrow \infty} \varphi(x)=\infty$ [13]. Clearly the inverse of $\varphi$ exists and is superadditive, strictly increasing and continuous, $\varphi^{-1}(0)=0$. Here $\varphi$ is said to be subadditive if $\varphi(s+t) \leq \varphi(s)+\varphi(t)$ for every $s, t \in[0, \infty)$, and $\varphi^{-1}$ is said to be superadditive if $\varphi^{-1}(s+t) \geq \varphi^{-1}(s)+\varphi^{-1}(t)$ for every $s, t \in[0, \infty)$. Functions like $\varphi(t)=t, \varphi(t)=\sqrt[n]{t}, \varphi(t)=\ln (1+t)$, are examples of elements in $\Phi$.

Although lower semi-continuity is important in this type of problem it is not essential. Here we will use more general functions, lower semi-continuous from above. A function $f: E \rightarrow \mathbb{R} \cup\{\infty\}$ is said to be lower semi-continuous from above at $x_{0}$ if $x_{n} \rightarrow x_{0}$ and $f\left(x_{1}\right) \geq f\left(x_{2}\right) \geq \cdots \geq f\left(x_{n}\right) \geq \cdots$ imply that $f\left(x_{0}\right) \leq \lim _{n \rightarrow \infty} f\left(x_{n}\right)[5]$.

\section{Ekeland-type variational principle}

In this section we present a generalization of Ekeland-Type variational principle for locally complete spaces and for lower semi-continuous from above functions.

Theorem 3.1. Let $(E, \tau)$ be a locally complete space and $f: E \rightarrow \mathbb{R} \cup\{\infty\}$ be a proper, lower semicontinuous from above and bounded below function. Let $\varphi$ be in $\Phi$ and $x_{0}$ be a point in Dom $(f)$, that is $f\left(x_{0}\right)<\infty$. Then for any Banach disk $B$ in $E$ such that $x_{0} \in E_{B}$ there exists $x^{*} \in E_{B}$ such that:

(a) $f\left(x^{*}\right)+\varphi\left(\rho_{B}\left(x^{*}-x_{0}\right)\right) \leq f\left(x_{0}\right)$ and

(b) $f\left(x^{*}\right)<f(x)+\varphi\left(\rho_{B}\left(x^{*}-x\right)\right)$ for all $x \in E_{B} \backslash\left\{x^{*}\right\}$.

Proof. Let $B$ be a Banach disk in $E$ such that $x \in\left(E_{B}, \rho_{B}\right)$ and let

$$
S(x)=\left\{y \in E_{B}: f(y)+\varphi\left(\rho_{B}(y-x)\right) \leq f(x)\right\} .
$$

Observe that $S(x)$ is nonempty and that if $y$ is in $S(x)$, then $S(y) \subset S(x)$, since for $z \in S(y)$, we have

$$
\begin{aligned}
\varphi\left(\rho_{B}(z-x)\right) & \leq \varphi\left(\rho_{B}(z-y)\right)+\varphi\left(\rho_{B}(y-x)\right) \\
& \leq f(y)-f(z)+f(x)-f(y)=f(x)-f(z) .
\end{aligned}
$$


Let $g: E_{B} \rightarrow \mathbb{R} \cup\{\infty\}$ be the function defined by,

$$
g(x)=\left\{\begin{array}{l}
f(x), x \in S\left(x_{0}\right) \\
\infty, x \in E_{B} \backslash S\left(x_{0}\right) .
\end{array}\right.
$$

Note that $g$ is both $\rho_{B}$-lower semi-continuous from above and bounded below.

Now, starting from any $x_{1}$ in $S\left(x_{0}\right)$ construct, inductively, $x_{k}$ in $S\left(x_{k-1}\right)$ such that,

$$
g\left(x_{k}\right) \leq \inf \left\{g(x): x \in S\left(x_{k-1}\right)\right\}+\frac{1}{k}
$$

since $x_{k} \in S\left(x_{k-1}\right)$, we obtain from the definition of $S\left(x_{k-1}\right)$,

$$
g\left(x_{k}\right)+\varphi\left(\rho_{B}\left(x_{k}-x_{k-1}\right)\right) \leq g\left(x_{k-1}\right)
$$

then $0 \leq \varphi\left(\rho_{B}\left(x_{k}-x_{k-1}\right)\right) \leq g\left(x_{k-1}\right)-g\left(x_{k}\right)$. So the bounded below sequence $\left(g\left(x_{k}\right)\right)$ is decreasing, that is, there is a real number $r$ such that

$$
r \leq g\left(x_{k}\right) \leq g\left(x_{k-1}\right) .
$$

So $g\left(x_{k}\right) \downarrow r$. Now let us prove that the sequence $\left(x_{k}\right)$ is $\rho_{B}$-Cauchy. By using the triangle inequality and the subadditivity of $\varphi$ we have for every $\delta>0$ that

$$
\begin{aligned}
& \varphi\left(\rho_{B}\left(x_{k}-x_{l}\right)\right) \\
\leq & \varphi\left(\rho_{B}\left(x_{k}-x_{k+1}\right)+\rho_{B}\left(x_{k+1}-x_{k+2}\right)\right)+\cdots+\left(\rho_{B}\left(x_{l-1}-x_{l}\right)\right) \\
\leq & \varphi\left(\rho_{B}\left(x_{k}-x_{k+1}\right)\right)+\varphi\left(\rho_{B}\left(x_{k+1}-x_{k+2}\right)\right)+\cdots+\varphi\left(\rho_{B}\left(x_{l-1}-x_{l}\right)\right) \\
\leq & g\left(x_{k}\right)-g\left(x_{k+1}\right)+g\left(x_{k+1}\right)-g\left(x_{k+2}\right)+\cdots+g\left(x_{l-1}\right)-g\left(x_{l}\right) \\
= & g\left(x_{k}\right)-g\left(x_{l}\right)<\delta \text { if } N(\delta) \leq k \leq l \text { for some } N(\delta) \text { in } \mathbb{N} .
\end{aligned}
$$

Since $\varphi^{-1}$ is continuous then for every $\epsilon>0$ there is a $\delta>0$ and therefore $N(\delta) \in \mathbb{N}$, such that if $N(\delta) \leq k \leq l$, then $g\left(x_{k}\right)-g\left(x_{l}\right)<\delta$ and $\varphi^{-1}\left(g\left(x_{k}\right)-\right.$ $\left.g\left(x_{l}\right)\right)<\epsilon$. So $\rho_{B}\left(x_{k}-x_{l}\right)<\varphi^{-1}\left(g\left(x_{k}\right)-g\left(x_{l}\right)\right)<\epsilon$ means that $\left(x_{k}\right)$ is $\rho_{B^{-}}$ Cauchy. So $E$ locally complete implies that there is $x^{*} \in E_{B}$ such that $\left(x_{k}\right)$ is $\rho_{B}$-convergent to $x^{*}$.

We still need to prove that (a) and (b) hold.

For (a) let $h_{n}: E_{B} \rightarrow \mathbb{R} \cup\{\infty\}$ be the function define by $h_{n}(x)=f(x)+$ $\varphi\left(\rho_{B}\left(x-x_{n}\right)\right)$, this functions are lower semi-continuous from above since it is the sum of $\left.f\right|_{E_{B}}$ which is lower semi-continuous from above added to a continuous function. Furthermore if $x_{k} \in S\left(x_{k-1}\right)$, then $f\left(x_{k-1}\right) \geq f\left(x_{k}\right)+$ $\varphi\left(\rho_{B}\left(x_{k}-x_{k-1}\right)\right)$ so adding $\varphi\left(\rho_{B}\left(x_{k-1}-x_{n}\right)\right)$ to both sides of the inequality we get

$$
\begin{aligned}
& f\left(x_{k-1}\right)+\varphi\left(\rho_{B}\left(x_{k-1}-x_{n}\right)\right) \\
\geq & f\left(x_{k}\right)+\varphi\left(\rho_{B}\left(x_{k}-x_{k-1}\right)\right)++\cdots+\varphi\left(\rho_{B}\left(x_{k-1}-x_{n}\right)\right) \\
\geq & f\left(x_{k}\right)+\varphi\left(\rho_{B}\left(x_{k}-x_{k-1}\right)++\cdots+\rho_{B}\left(x_{k-1}-x_{n}\right)\right) \\
\geq & f\left(x_{k}\right)+\varphi\left(\rho_{B}\left(x_{k}-x_{k-1}+x_{k-1}-x_{n}\right)\right) \\
\geq & f\left(x_{k}\right)+\varphi\left(\rho_{B}\left(x_{k}-x_{n}\right)\right) .
\end{aligned}
$$


Now we have that $\left(h_{n}\left(x_{k}\right)\right)$ is a decreasing sequence for every $n \in \mathbb{N}$, and by the lower semi-continuity from above $h_{n}\left(x^{*}\right) \leq \lim _{k \rightarrow \infty} h_{n}\left(x_{k}\right)$ then

$$
\begin{aligned}
f\left(x^{*}\right)+\varphi\left(\rho_{B}\left(x^{*}-x_{n}\right)\right) & \leq \lim _{k \rightarrow \infty}\left[f\left(x_{k}\right)+\varphi\left(\rho_{B}\left(x_{k}-x_{n}\right)\right]\right. \\
& \leq f\left(x_{k}\right)+\varphi\left(\rho_{B}\left(x_{k}-x_{n}\right)\right) \text { for every } n, k \in \mathbb{N} \cup\{0\} .
\end{aligned}
$$

So taking by taking $k=n$ we have $x^{*} \in S\left(x_{k}\right)$ for every $k \in \mathbb{N}$, in particular $x^{*} \in S\left(x_{0}\right)$ and we have proved (a).

Now we will prove that $S\left(x^{*}\right)=\left\{x^{*}\right\}$. Suppose that $x \in S\left(x^{*}\right) \subset S\left(x_{k-1}\right)$ then

$$
f\left(x^{*}\right) \leq f\left(x_{k}\right) \leq \inf _{x \in S\left(x_{k-1}\right)} f(x)+\frac{1}{k} \leq f(x)+\frac{1}{k} \text { for every } k \in \mathbb{N} .
$$

So $f\left(x^{*}\right) \leq f(x)$ and then

$$
f\left(x^{*}\right) \leq f\left(x^{*}\right)+\varphi\left(\rho_{B}\left(x^{*}-x\right)\right) \leq f(x)+\varphi\left(\rho_{B}\left(x^{*}-x\right)\right) \leq f\left(x^{*}\right)
$$

and we get $\varphi\left(\rho_{B}\left(x^{*}-x\right)\right)=0$. Now by the continuity of $\varphi^{-1}$, and the fact that $\varphi(0)=0$, we have that $\rho_{B}\left(x^{*}-x\right)=0$ which means that $x^{*}=x$ then $S\left(x^{*}\right)=\left\{x^{*}\right\}$ if $x \in E_{B}, x \neq x^{*}$, so we get inequality (b) in the theorem. If $x \in E \backslash E_{B}$, it is clear that the inequality holds, since $\rho_{B}(x)=\infty$.

Remark 3.2. A sequence $\left(x_{n}\right)$ is said to be locally convergent or Mackey convergent to an element $x$ of $E$, if there exists a Banach disc $B$ in $E$ such that the sequence converges in $E_{B}$ with respect to $\rho_{B}$. For more details see PerezCarreras and Bonet [11]. With this in mind it is easy to generalized the previous theorem to locally lower semi-continuous from above functions which are lower semi-continuous from above functions that, for each $x$ in $E$ if $\left(x_{n}\right)$ is locally convergent to $x$ and $f\left(x_{k}\right)$ is decreasing, then $f(x) \leq \lim _{n \rightarrow \infty} f\left(x_{n}\right)$.

\section{Equivalences}

Now we will give an equivalence between a Caristi-Kirk type fixed point theorem, Takahashi type minimization theorem, an equilibrium version of Ekelandtype Variational Principle and Theorem 3.1 for Qiu's perturbations [13], lower semi-continuous from above functions [5] and in the setting of locally complete spaces.

Theorem 4.1. Let $(E, \tau)$ be a locally complete space, $f: E \rightarrow \mathbb{R}$ be a lower semicontinuous from above, bounded below function and let $\varphi$ be in $\Phi$. Then the following statements are equivalent to Theorem 3.1:

(i) (Caristi-Kirk type fixed point theorem). Let $2^{E}$ denotes the set of all subsets of $E$ and $T: E \rightarrow 2^{E}$ be a multivalued map with nonempty values. If there exists a Banach disk $B$ in $E$ such that for all $x \in E_{B}$ and $y \in T x$ we have

$$
\varphi\left(\rho_{B}(x-y)\right) \leq f(x)-f(y)
$$


holds, then $T$ has a stationary point in $E_{B}$, that is, there exists $x^{*} \in E_{B}$ such that $\left\{x^{*}\right\}=T x^{*}$.

(ii) (Takahashi type minimization theorem). Assume that for each $x^{\prime} \in E$ with $\inf _{z \in E} f(z)<f\left(x^{\prime}\right)$ there exists a Banach disk $B$ in $E$ such that $x^{\prime} \in E_{B}$ and there exists $x \in E_{B}-\left\{x^{\prime}\right\}$ such that $\varphi\left(\rho_{B}\left(x^{\prime}-x\right)\right) \leq f\left(x^{\prime}\right)-f(x)$. Then there exists $x^{*} \in E_{B}$ such that $f\left(x^{*}\right)=\inf _{y \in E_{B}} f(y)$.

(iii) (Equilibrium version of Ekeland-type Variational Principle). Let $F$ : $E \times E \rightarrow \mathbb{R}$ such that

1. For all $x, y, z \in E, F(x, z) \leq F(x, y)+F(y, z)$.

2. For each fixed $x \in E, F(x, \cdot): E \rightarrow \mathbb{R}$ is lower semi continuous from above.

3. There exists $x^{\prime} \in E$ such that $\inf _{x \in E} F\left(x^{\prime}, x\right)>-\infty$.

Then there exists a Banach disk $B$ in $E, x^{\prime} \in E_{B}$ and $x^{*} \in E_{B}$ such that

(a) $F\left(x^{\prime}, x^{*}\right)+\varphi\left(\rho_{B}\left(x^{\prime}-x^{*}\right)\right) \leq 0$,

(b) $F\left(x^{*}, x\right)+\varphi\left(\rho_{B}\left(x^{*}-x\right)\right)>0$ for all $x \in E_{B} \backslash\left\{x^{*}\right\}$.

Proof. We will prove Theorem $3.1 \Longrightarrow$ (i) $\Longrightarrow$ (ii) $\Rightarrow$ (iii) $\Longrightarrow$ Theorem 3.1.

From Theorem 3.1 part (b) there exists $x^{*} \in E_{B}$ such that

$$
f\left(x^{*}\right)-f(x)<\varphi\left(\rho_{B}\left(x^{*}-x\right)\right) \text { for all } x \in E_{B} \backslash\left\{x^{*}\right\} .
$$

We claim that $\left\{x^{*}\right\}=T x^{*}$. Otherwise if $y \in T x^{*} \backslash\left\{x^{*}\right\}$ from (1) we have

$$
\varphi\left(\rho_{B}\left(x^{*}-y\right)\right) \leq f\left(x^{*}\right)-f(y)
$$

which contradicts the previous inequality. Note that also from inequality (1), for all $x \in E_{B}$ and $y \in T x$, we must have $\rho_{B}(x-y)<\infty$, this means $y \in E_{B}$, i.e., $T x \subset E_{B}$ for all $x \in E_{B}$.

$$
\begin{aligned}
& (\mathrm{i}) \Longrightarrow(\text { ii }) \text {. Define } T: E \rightarrow 2^{E} \text { as } \\
& T(x)= \begin{cases}\left\{y \in E_{B}: \varphi\left(\rho_{B}(x-y)\right) \leq f(x)-f(y)\right\} & \text { if } x \in E_{B} . \\
E & \text { if } x \in E \backslash E_{B}\end{cases}
\end{aligned}
$$

Note that $T$ satisfies inequality (1). Then by (i) there exists $x^{*} \in E_{B}$ such that $\left\{x^{*}\right\}=T x^{*}$. Now by assumption for each $x^{\prime} \in E_{B}$ there exists $x \in E_{B} \backslash\left\{x^{\prime}\right\}$, we have $x \in T x^{\prime}$ and then $T x^{\prime} \backslash\left\{x^{\prime}\right\} \neq \emptyset$ whenever $\inf _{z \in E} f(z)<$ $f\left(x^{\prime}\right)$ hence we must have $\inf _{z \in E_{B}} f(z)=f\left(x^{*}\right)$.

(ii) $\Longrightarrow$ (iii). Define a function $f: E \rightarrow \mathbb{R}$ by $f(x)=F\left(x^{\prime}, x\right)$ for all $x \in E$ where $x^{\prime}$ is the element given in condition (iii-3). Then we have $\inf _{x \in E} F\left(x^{\prime}, x\right)>-\infty$ which means that $f$ is bounded below and by (iii-2), $f$ is proper lower semicontinuous from above. Let $B \subset E$ be a Banach disk such that $x^{\prime} \in E_{B}$. Now suppose that in (iii), (3-b) does not hold. Then for all $x \in E_{B}$ there exists $y \in E_{B} \backslash\{x\}$ and $F(x, y)+\varphi\left(\rho_{B}(x-y)\right) \leq 0$. By condition (iii-1), we have $F\left(x^{\prime}, y\right)-F\left(x^{\prime}, x\right) \leq F(x, y)$ so using this and the previous inequality:

$$
F\left(x^{\prime}, y\right)-F\left(x^{\prime}, x\right)+\varphi\left(\rho_{B}(x-y)\right) \leq F(x, y)+\varphi\left(\rho_{B}(x-y)\right) \leq 0
$$

that is, for all $x \in E_{B}$ there exists $y \in E_{B}-\{x\}$ and

$f(y)-f(x)+\varphi\left(\rho_{B}(x-y)\right) \leq 0$, or equivalently $\varphi\left(\rho_{B}(x-y)\right) \leq f(x)-f(y)$. 
Then by (ii), there exists $x^{*} \in E_{B}$ such that $f\left(x^{*}\right) \leq f(z)$ for all $z \in E_{B}$. By substituting $x$ by $x^{*}$ in inequality (2), we obtain that there exists $y \in E_{B} \backslash\left\{x^{*}\right\}$ and $\varphi\left(\rho_{B}\left(x^{*}-y\right)\right) \leq f\left(x^{*}\right)-f(y)$. Now since $\rho_{B}$ is a norm in $E_{B}, \rho_{B}\left(x^{*}-y\right)>0$, and then $f(y)<f\left(x^{*}\right)$ which is a contradiction.

Finally let us prove that (iii) $\Rightarrow$ Theorem 3.1. Define $F: E \times E \rightarrow \mathbb{R}$ as $F(x, y)=f(y)-f(x)$ for all $x, y \in E$, with $x^{\prime} \in d o m(f)$. Then by hypothesis, $F$ satisfies all the conditions of (iii). Then (iii) implies the existence of $x^{*} \in E_{B}$ such that (a) and (b) hold.

\section{References}

[1] S. Al-Homidan, Q. H. Ansari, and J.-C. Yao, Some generalizations of Ekeland-type variational principle with applications to equilibrium problems and fixed point theory, Nonlinear Anal. 69 (2008), no. 1, 126-139.

[2] M. Bianchi, G. Kassey, and R. Pini, Existence of equilibria via Ekeland's principle, J. Math. Anal. Appl. 284 (2003), 690-697.

[3] C. Bosch, A. García, and C. L. García, An extension of Ekeland's variational principle to locally complete spaces, J. Math. Anal. Appl. 328 (2007), 106-108.

[4] C. Bosch, A. García, C. Gómez-Wulschner, and S. Hernández-Linares, Equivalents to Ekeland's variational principle in locally complete spaces, Sci. Math. Japn. 72 (2010), no. 3, 283-287.

[5] Y. Chen, Y. J. Cho, and L. Yang, Note on the results with lower semi-continuity, Bull. Korean Math. Soc. 39 (2002), no. 4, 535-541.

[6] I. Ekeland, On the variational principle, J. Math. Anal. Appl. 47 (1974), 324-353.

[7] J. X. Fang, The variational principle and fixed point theorems in certain topological spaces, J. Math. Anal. Appl. 202 (1996), 398-412.

[8] A. H. Hamel, Phelp's lemma, Danes'drop theorem and Ekeland's principle in locally convex spaces, Proc. Amer. Math. Soc. 131 (2003), no. 10, 3025-3038.

[9] _ Equivalents to Ekeland's variational principle in uniform spaces, Nonlinear Anal. 62 (2005), no. 5, 913-924.

[10] H. Jarchow, Locally Convex Spaces, B. G. Teubner, Stuttgart, 1981.

[11] P. Pérez-Carreras and J. Bonet, Barrelled Locally Convex Spaces, North-Holland, Amsterdam, 1987.

[12] J. H. Qiu, Local completeness and drop theorem, J. Math. Anal. Appl. 266 (2002), no. 2, 288-297.

[13] — Ekeland's variational principle in locally complete spaces, Math. Nachr. 257 (2003), 55-58.

[14] Local completeness, drop theorem and Ekeland's variational principle, J. Math. Anal. Appl. 311 (2005), no. 1, 23-39.

CARlos Bosch

Departmento de Matemáticas

instituto Tecnológico Autónomo de México:

Rio Hondo \# 1, Del. Alvaro Obregón, 01000 México D.F., México

E-mail address: bosch@itam.mx

RENÉ LEAL

Departmento de Matemáticas

Instituto Tecnológico Autónomo de México:

Rio Hondo \# 1, Del. Alvaro Obregón, 01000 México D.F., México

E-mail address: rene.leal.12@gmail.com 